\title{
An Interpolation in Polygonal Networks of Resistors
}

Je-Young Choi

\author{
Department of Smart IT, U1 University, Asan 31415, Korea; jychoi@u1.ac.kr
}

\begin{abstract}
Objectives: Ladder networks of resistors have been discussed extensively. This paper considers polygons of resistors where the resistors on sides are different from those on spokes. The objective is to find how their physical quantities depend on the parity of the number of the sides. Methods: We calculate attenuations, nodal potentials, and input impedances when a voltage source is connected between a node and the center. We introduce a continuous parameter $\rho$ in equivalent ladder networks where $\rho=1$ and $\rho=2$ correspond to odd and even numbers of sides, respectively. Findings: Attenuations, nodal potentials, and input impedances are expressed in terms of the Chebyshev polynomials of the second kind or the Fibonacci polynomials. The results depend on the parity of the number of sides. The case $\rho=0$ interpolates the case with the odd numbers of sides. Application: The method presented in this document can be applicable to networks with inhomogeneous resistances around the sides.
\end{abstract}

Keywords: Chebychev Polynomials, Electric Circuit, Fibonacci polynomials, Interpolation, Polygonal Network

\section{Introduction}

Ladder networks consist of passive elements like resistors, capacitors, and inductors and have applications in filters and transmission lines. It is well known that the Fibonacci numbers appear in a ladder network of equal resistors ${ }^{1}$. Physical quantities such as input impedances (or equivalent resistances), attenuations, and nodal potentials in a ladder of resistors which is homogeneous along the ladder, that is, has identical series and identical parallel (shunt) resistors, respectively, have been calculated. They are expressed in terms of Morgan-Voyce polynomials which have been studied extensively ${ }^{2-7}$.

Fibonacci numbers appear also in a polygon of resistors where equal resistors are connected along sides and spokes ${ }^{\circ}$. This can be understood since such a polygon of resistors can be deformed to a ladder network $\frac{9-10}{}$. The purpose of this article is to determine physical quantities of the polygons where the resistors on the sides are different from those on the spokes.
Their expressions depend on the parity of the number of the sides of the polygons and are expressed in terms of the Chebyshev polynomials of the second kind or equivalently in terms of the Fibonacci polynomials. Finally we find a polygon of resistors interpolating the ones with odd numbers of sides.

\section{Polygon of Resistors}

We consider a polygon of resistors with $n(=2 m)$ sides $^{9}$ where the resistors on the sides have the resistance $2 r_{\mathrm{s}}$ while the resistors on the spokes have $2 r_{\mathrm{p}}$. Figure 1 depicts an odd $n$ case whereas Figure 2 an even $n$ case. We removed a resistor joining the $\oplus=m$ and $\Theta$ nodes following PareraLope $z^{10}$ in order to compare easily with results of ladder networks. By the reflection symmetry about the line joining $\oplus$ and $\Theta$ nodes the potentials $V_{i}$ and $V_{i^{\prime}}$ at the nodes $i$ and $i^{\prime}$ respectively are the same for each $i=0, \ldots, m-1$. When $n$ is odd, $V_{0}=V_{0^{\prime}}$ so that the resistor joining the two nodes 0 and $0^{\prime}$ can be eliminated. 


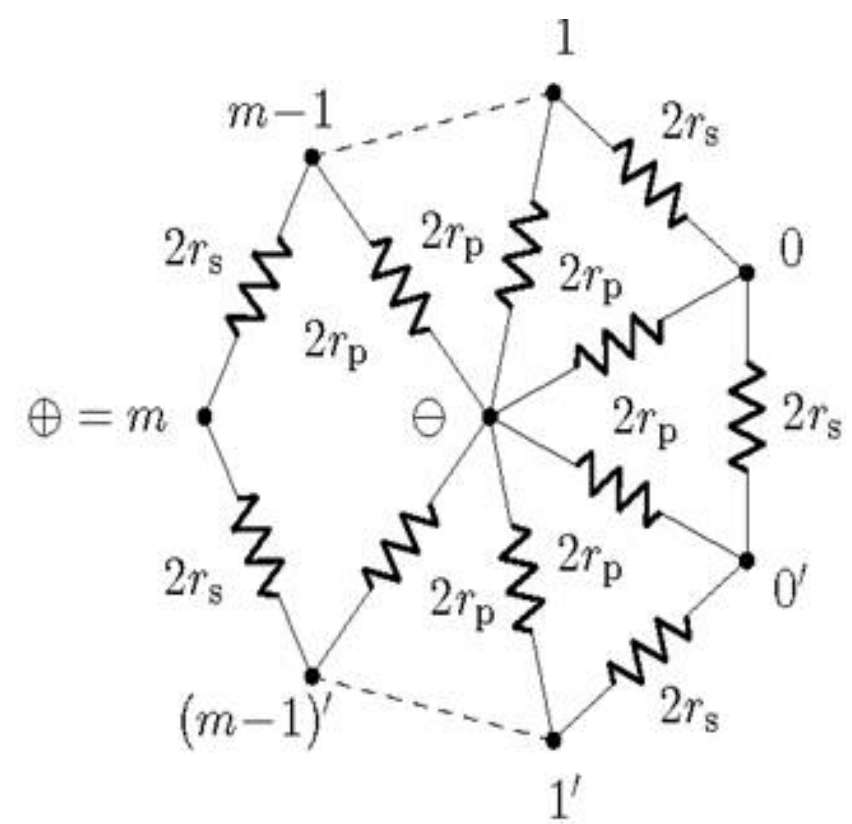

Figure 1. A polygon of resistors. Here the number of sides is $n=2 m+1$.

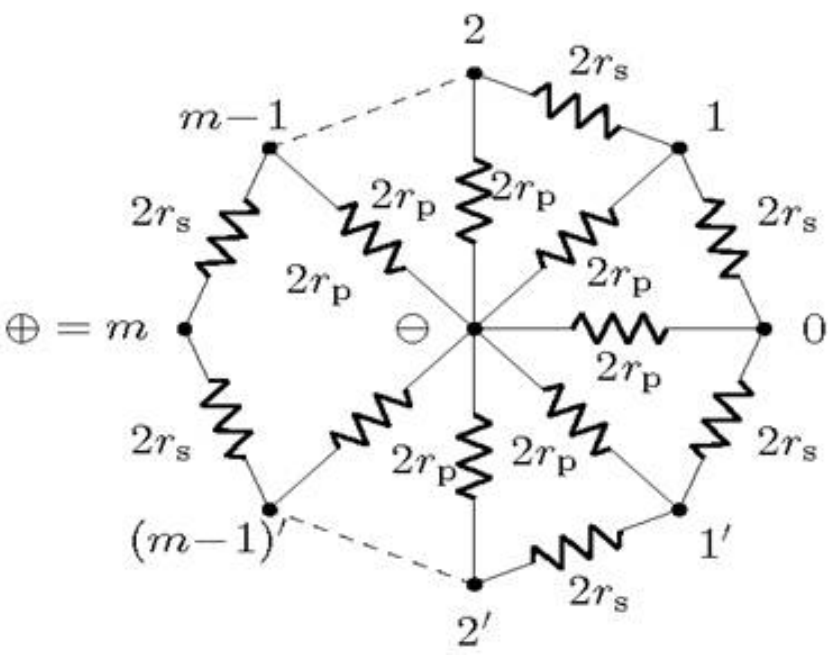

Figure 2. A polygon of resistors. Here $n=2 m$.

Following Sidhu ${ }^{9}$ and Pareta-Lopez ${ }^{10}$ we transform the polygon of resistors in Figure 1 or Figure 2 into a ladder network of resistors in Figure 3. In the ladder network equivalents series resistors have resistance $r_{\mathrm{s}}$ while parallel resistors have resistance $r_{\mathrm{p}}$ except the rightmost one with $\rho r_{\mathrm{p}}$ for a continuous parameter $\rho$ in Figure 3 where $\rho=1$ for odd $n=2 m+1$ and $\rho=2$ for even $n=2 m$. We see that the nodal potential $V_{i}$ at the node $i$ in Figure 1 is equal to that in Figure 3 with $\rho=1$ and that the input impedance $R_{m}$ between nodes $\oplus=m$ and $\ominus$ in Figure 1 is equal to that in the ladder network shown in Figure 3 with $\rho=1$. The same is true for Figure 2 and Figure 3 with $\rho=2$ for even $n$ case.

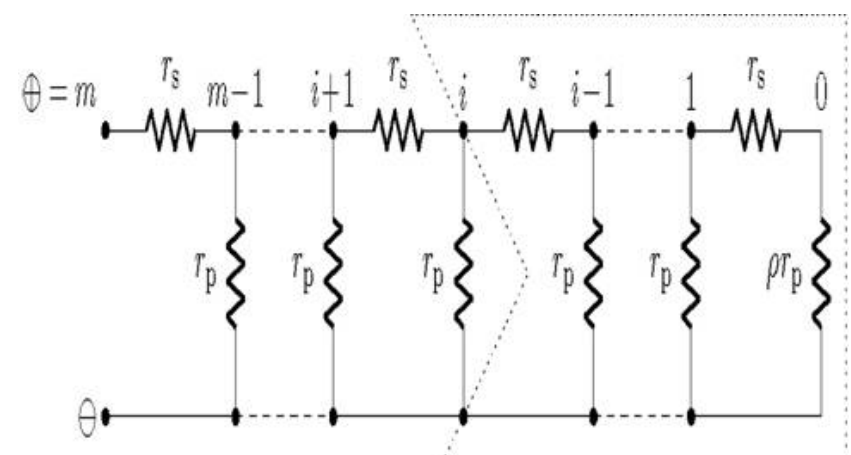

Figure 3. The ladder equivalent. The equivalent of the polygon with $n=2 m+1$ or $n=2 m$ includes the equivalent of the $2 i+1$ or $2 i$ sided polygon surrounded by the dotted line for $0<i<m$, respectively.

We apply a d.c. voltage source to the network so that $V_{\oplus}=\mathcal{E}, V_{\odot}=0$. The Kirchhoff's current law at the node $i$ gives

$$
\frac{V_{i+1}-V_{i}}{r_{s}}=\frac{V_{i}-V_{i-1}}{r_{s}}+\frac{V_{i}}{r_{p}}
$$

or

$$
V_{i+1}=(x+2) V_{i}-V_{i-1},
$$

which can be written in matrix form as

$$
\left(\begin{array}{c}
V_{i+1} \\
V_{i}
\end{array}\right)=T\left(\begin{array}{c}
V_{i} \\
V_{i-1}
\end{array}\right),
$$

where $T$ is a transfer matrix given by

$$
T=\left(\begin{array}{cc}
x+2 & -1 \\
1 & 0
\end{array}\right)
$$

with $x=r_{\mathrm{s}} / r_{\mathrm{p}}$ (see Trzaska $\left.{ }^{11}\right)$. The characteristic equation for $T$ is

$$
0=\operatorname{det}(\lambda I-T)=\lambda^{2}-(x+2) \lambda+1 .
$$

Then the Cayley-Hamilton theorem gives

$$
T^{2}=(x+2) T-I .
$$

If we let

$$
T^{i}=q_{i} T-q_{i-1} I,
$$

then we find the recurrence relation for $q_{i}$

$$
q_{i+1}=(x+2) q_{i}-q_{i-1}, \quad q_{1}=1, q_{0}=0 .
$$

Comparing with the definition of Chebyshev polynomials of the second kind

$$
W_{i}(\cos k)=\frac{\sin (i+1) k}{\sin k}
$$

which satisfy the recurrence relation

$$
\begin{aligned}
& W_{i+1}(x)=2 x W_{i}(x)-W_{i-1}(x), \\
& W_{0}(x)=1, \quad W_{-1}(x)=0,
\end{aligned}
$$

we obtain

$$
q_{i}=W_{i-1}(x / 2+1) .
$$


Iterating equation (3) gives

$$
\begin{aligned}
& \left(\begin{array}{l}
V_{i+1} \\
V_{i}
\end{array}\right)=T^{i}\left(\begin{array}{l}
V_{1} \\
V_{0}
\end{array}\right) \\
& =\left(q_{i} T-q_{i-1} I\right)\left(\begin{array}{l}
V_{1} \\
V_{0}
\end{array}\right)
\end{aligned}
$$

from which we find the recurrence relation for $V_{i}$

$$
V_{i}=q_{i} V_{1}-q_{i-1} V_{0} \text {. }
$$

Since we see $V_{1}: V_{0}=\left(r_{\mathrm{s}}+\rho r_{\mathrm{p}}\right): \rho r_{\mathrm{p}}$, or for $\rho \neq 0$ from Figure 3

$$
V_{1}=\left(\frac{x}{\rho}+1\right) V_{0},
$$

we have the attenuation (or transfer ratio) at the node $i$

$$
\begin{aligned}
& A_{i} \equiv V_{i} / V_{0}=\left(\frac{x}{\rho}+1\right) q_{i}-q_{i-1} \\
& =\frac{q_{i+1}+(\rho-2) q_{i}-(\rho-1) q_{i-1}}{\rho},
\end{aligned}
$$

where equation (8) has been used. The first equality in equation (15) in the case of $\rho=1$ was obtained by Trzaska. ${ }^{11}$ Letting $V_{\oplus}=V_{m}=\mathcal{E}$, we have

$$
\mathcal{E}=\frac{q_{m+1}+(\rho-2) q_{m}-(\rho-1) q_{m-1}}{\rho} V_{0}
$$

and obtain the result

$$
\frac{V_{i}}{\mathcal{E}}=\frac{q_{i+1}+(\rho-2) q_{i}-(\rho-1) q_{i-1}}{q_{m+1}+(\rho-2) q_{m}-(\rho-1) q_{m-1}} \quad(i=0, \ldots, m) .
$$

In view of equation (14), we see that equation (17) holds also at $i=0$.

As shown in Figure 3 the ladder equivalent of the $n$-sided polygon with $n=2 m+1$ or $n=2 m$ includes the $(2 i+1)$ - or $2 i$ - sided polygon equivalent for $0<i<m$, respectively. In order to evaluate the input impedance $R_{i}$, we use the proportionality

$$
\left(V_{i+1}-V_{i}\right): V_{i}=r_{\mathrm{s}}:\left(r_{\mathrm{p}} \| R_{i}\right),
$$

where we have used the operator $\|$ defined by

$$
r_{1} \| r_{2}=\frac{r_{1} r_{2}}{r_{1}+r_{2}}
$$

for the parallel combination of resistors. Solving for $R_{i}$ yields

$$
\frac{R_{i}}{r_{p}}=\frac{x V_{i}}{V_{i+1}-(x+1) V_{i}},
$$

which becomes, due to equations (15) and (8),

$$
\begin{gathered}
\frac{R_{i}}{r_{p}}=\frac{x\left[q_{i+1}+(\rho-2) q_{i}-(\rho-1) q_{i-1}\right]}{q_{i+2}+(\rho-2) q_{i+1}-(\rho-1) q_{i}-(x+1)\left[q_{i+1}+(\rho-2) q_{i}-(\rho-1) q_{i-1}\right]} \\
=\frac{q_{i+1}+(\rho-2) q_{i}-(\rho-1) q_{i-1}}{q_{i}+(\rho-1) q_{i-1}}
\end{gathered}
$$

which can also be read off from a result by Hong and Choi. $\frac{12}{}$ The resulting expressions for $A_{i}, V_{i} / \mathcal{E}$ and $R_{i} / r_{\mathrm{p}}$ depend on the value of $\rho$.

\subsection{Odd $n$ Case}

For $n=2 m+1$ we have $\rho=1$. Then we find the attenuation

$$
A_{i}=q_{i+1}-q_{i}
$$

and the nodal potentials

$$
\frac{V_{i}}{\mathcal{E}}=\frac{q_{i+1}-q_{i}}{q_{m+1}-q_{m}}
$$

for $i=0, \ldots, m$. The input impedance is given for $i>0$ by

$$
\frac{R_{i}}{r_{p}}=\frac{q_{i+1}-q_{i}}{q_{i}} .
$$

The numerator $b_{i}(x) \equiv q_{i+1}-q_{i}$ and the denominator $B_{i-1}(x) \equiv q_{i}$ of $R_{i} / r_{\mathrm{p}}$ are known as the Morgan-Voyce polynomials,,${ }^{2,4}$ so that

$A_{i}=b_{i}(x), V_{i} / \mathcal{E}=b_{i}(x) / b_{m}(x), R_{i} / r_{\mathrm{p}}=b_{i}(x) / B_{i-1}(x)$.

But due to equation (11) they are written in terms of Chebyshev polynomials of the second kind as follows

$$
\begin{aligned}
& B_{i}(x)=W_{i}(x / 2+1), \\
& b_{i}(x)=W_{i}(x / 2+1)-W_{i-1}(x / 2+1)
\end{aligned}
$$

as was obtained by Mowery ${ }^{\underline{13}}$ and Trzaska. ${ }^{11}$

It was also proved that $t^{3}, \underline{4}$

$$
\begin{aligned}
& B_{i-1}(x)=\frac{1}{\sqrt{x}} F_{2 i}(\sqrt{x}), \\
& b_{i}(x)=\frac{1}{\sqrt{x}}\left[F_{2 i+2}(\sqrt{x})-F_{2 i}(\sqrt{x})\right] \\
& =F_{2 i+1}(\sqrt{x}),
\end{aligned}
$$

where $F_{i}(x)$ are the Fibonacci polynomials defined by

$$
F_{i}(x)=x F_{i-1}(x)+F_{i-2}(x), \quad F_{1}(x)=1, F_{0}(x)=0 .
$$

Hence $A_{i}, V_{i} / \mathcal{E}$, and $R_{i} / r_{\mathrm{p}}$ can be expressed in terms of the Fibonacci polynomials:

$$
\begin{aligned}
& A_{i}=F_{2 i+1}(\sqrt{x}), \\
& \frac{V_{i}}{\mathcal{E}}=\frac{F_{2 i+1}(\sqrt{x})}{F_{2 m+1}(\sqrt{x})}, \\
& \frac{R_{i}}{r_{p}}=\frac{\sqrt{x} F_{2 i+1}(\sqrt{x})}{F_{2 i}(\sqrt{x})} .
\end{aligned}
$$

\subsection{Even $\boldsymbol{n}$ Case}

When $n=2 m$, then $\rho=2$ and we have the attenuation

$$
A_{i}=\frac{1}{2}\left(q_{i+1}-q_{i-1}\right)
$$




$$
=\frac{1}{2}\left[W_{i}(x / 2+1)-W_{i-2}(x / 2+1)\right]
$$

and the nodal potentials

$$
\begin{aligned}
& \frac{V_{i}}{\mathcal{E}}=\frac{q_{i+1}-q_{i-1}}{q_{m+1}-q_{m-1}} \\
& =\frac{W_{i}(x / 2+1)-W_{i-2}(x / 2+1)}{W_{m}(x / 2+1)-W_{m-2}(x / 2+1)} .
\end{aligned}
$$

For $i>0$, we have

$$
\begin{aligned}
& \frac{R_{i}}{r_{p}}=\frac{q_{i+1}-q_{i-1}}{q_{i}+q_{i-1}} \\
& =\frac{W_{i}(x / 2+1)-W_{i-2}(x / 2+1)}{W_{i-1}(x / 2+1)+W_{i-2}(x / 2+1)} .
\end{aligned}
$$

Using the Fibonacci polynomials, they are given by

$$
\begin{aligned}
& A_{i}=\frac{1}{2}\left[F_{2 i+1}(\sqrt{x})+F_{2 i-1}(\sqrt{x})\right], \\
& \frac{V_{i}}{\mathcal{E}}=\frac{F_{2 i+1}(\sqrt{x})+F_{2 i-1}(\sqrt{x})}{F_{2 m+1}(\sqrt{x})+F_{2 m-1}(\sqrt{x})}, \\
& \frac{R_{i}}{r_{p}}=\frac{\sqrt{x}\left[F_{2 i+1}(\sqrt{x})+F_{2 i-1}(\sqrt{x})\right]}{F_{2 i}(\sqrt{x})+F_{2 i-2}(\sqrt{x})} .
\end{aligned}
$$

\subsection{Interpolation Between Odd $\boldsymbol{n}$ Cases}

The expressions for even $n$ cases have different forms from those for odd $n$ cases. We can find expressions interpolating odd $n$ cases by choosing $\rho=0$ with the corresponding polygon of resistors depicted in Figure 4. Then $V_{0}=0$ and equation (15) cannot be used. But from equation (13) we have

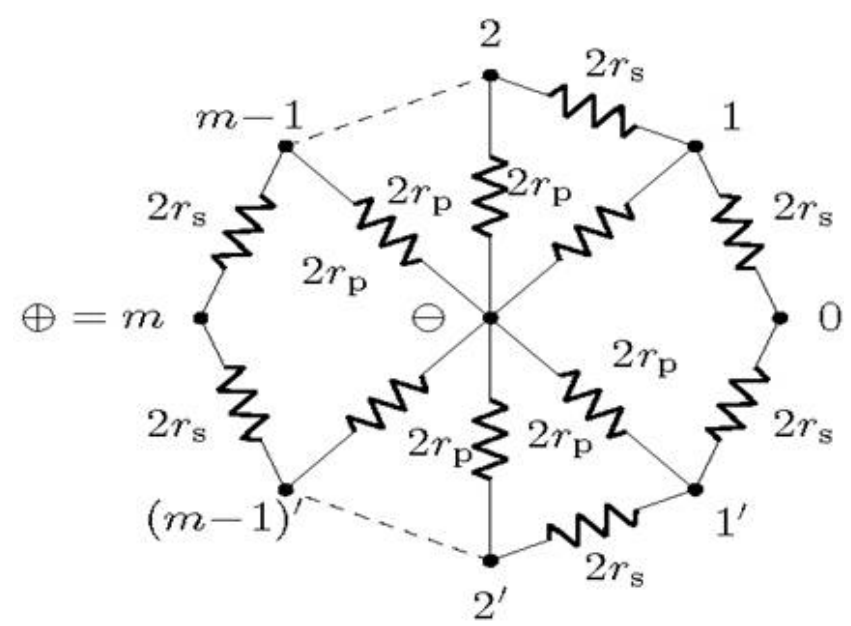

Figure 4. A polygon of resistors interpolating polygons with odd numbers of sides.

$$
V_{i}=q_{i} V_{1}=W_{i-1}(x / 2+1) V_{1}
$$

or

$$
\frac{V_{i}}{\mathcal{E}}=\frac{W_{i-1}(x / 2+1)}{W_{m-1}(x / 2+1)} .
$$

From equation (21), we find

$$
\begin{aligned}
& \frac{R_{i}}{r_{p}}=\frac{q_{i+1}-2 q_{i}+q_{i-1}}{q_{i}-q_{i-1}} \\
& =\frac{x q_{i}}{q_{i}-q_{i-1}} \\
& =\frac{x W_{i-1}(x / 2+1)}{W_{i-1}(x / 2+1)-W_{i-2}(x / 2+1)} .
\end{aligned}
$$

In terms of the Fibonacci polynomials, they are expressed as

$$
\begin{aligned}
& \frac{V_{i}}{\mathcal{E}}=\frac{F_{2 i}(\sqrt{x})}{F_{2 m}(\sqrt{x})}, \\
& \frac{R_{i}}{r_{p}}=\frac{\sqrt{x} F_{2 i}(\sqrt{x})}{F_{2 i-1}(\sqrt{x})}
\end{aligned}
$$

which interpolate the results given by equation (29) for the polygons with $2 i+1$ and $2 i-1$ sides.

\section{Concluding Remarks}

We have considered polygons of resistors and their equivalent ladder networks. The attenuations, nodal potentials, and input impedances are written in terms of the Chebyshev polynomials of the second kind or in terms of the Fibonacci polynomials and depend on the parity of the number of sides of the polygon. The ladder networks considered have a continuous parameter $\rho$. André-Jeannin ${ }^{6}$ introduced generalized polynomials $P_{n}^{(r)}(x)$ which are found to satisfy

$$
P_{n}^{(r)}(2 \omega-2)=W_{n}(\omega)+(r-1) W_{n-1}(\omega) .
$$

It follows from equation (11) that equations (15), (17), and (21) can be written, respectively, as

$$
\begin{aligned}
& A_{i}=\frac{P_{i}^{(\rho)}(x)-P_{i-1}^{(\rho)}(x)}{\rho}, \\
& \frac{V_{i}}{\mathcal{E}}=\frac{P_{i}^{(\rho)}(x)-P_{i-1}^{(\rho)}(x)}{P_{m}^{(\rho)}(x)-P_{m-1}^{(\rho)}(x)}, \\
& \frac{R_{i}}{r_{p}}=\frac{P_{i}^{(\rho)}(x)-P_{i-1}^{(\rho)}(x)}{P_{i-1}^{(\rho)}(x)},
\end{aligned}
$$


so that the polynomials $P_{n}^{(r)}(x)$ find real applications. In fact, due to equations (26) and (27), $P_{n}^{(r)}(x)$ can be written in terms of the Fibonacci polynomials

$$
P_{n}^{(r)}(x)=\frac{1}{\sqrt{x}}\left[F_{2 n+2}(\sqrt{x})+(r-1) F_{2 n}(\sqrt{x})\right] \text {. }
$$

Finally we note that physical quantities in a ladder network with inhomogeneous resistances, for example, with exponentially varying resistances along the ladder can be calculated and will be an interesting system to be analyzed.

\section{References}

1. Basin SL. The Fibonacci sequence As It Appears in Nature. Fibonacci Quarterly. 1963 Feb; 1(1):53-6.

2. Morgan-Voyce AM. Ladder-Network Analysis Using Fibonacci Numbers. IRE Transactions on Circuit Theory. 1959 Sep; 6(3):321-2. Crossref.

3. Swamy MNS. Further Properties of Morgan-Voyce Polynomials. Fibonacci Quarterly. 1968 Apr; 6(2):167-75.

4. HoggattJr VE, Bicknell M. A Primer for the Fibonacci Numbers: Part XIV. Fibonacci Quarterly. 1974 Apr; 12(2):147-56.

5. Ferri G, Faccio M, D'Amico A. Fibonacci Numbers and Ladder Network Impedance. Fibonacci Quarterly. 1992 Feb; 30(1): 62-7.
6. André-Jeannin R. A Generalization of Morgan-Voyce Polynomials. Fibonacci Quarterly. 1994 Jun-Jul; 32(3): 228-31.

7. Swamy MNS. Generalizations of Modified Morgan-Voyce Polynomials. Fibonacci Quarterly. 2000 Feb; 38(1): 8-16.

8. March RH. Polygons of resistors and convergent series. American Journal of Physics. 1993 Oct; 61(10): 900-1. Crossref.

9. Sidhu SS. Polygons of unequal resistors. American Journal of Physics. 1994 Sep; 62(9): 815-6. Crossref.

10. Parera-Lopez JJ. T-iterated electrical networks and numerical sequences. American Journal of Physics. 1997 May; 65(5): 437-9. Crossref.

11. Trzaska ZW. Numerical triangle, Fibonacci sequence and ladder networks: Some further results. Applied Mathematics Letters. 1993 Jul; 6(4): 55-61. Crossref.

12. Hong S-I, Choi J-Y. A New Method of Calculating Input Impedances of Inhomogeneous Ladder Networks. Journal of the Korean Physical Society. 2012 Jun; 60(11): 1851-6. Crossref.

13. Mowery VO. Fibonacci Numbers and Tchebycheff Polynomials in Ladder Networks. IRE Transactions on Circuit Theory. 1961 Jun; 8(2): 167-8. Crossref. 